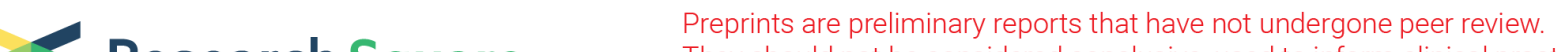 Research Square They should not be considered conclusive, used to inform clinical practice, or referenced by the media as validated information.
}

\section{Molecular evidence of rat bocavirus among rodents in Peninsular Malaysia.}

Siti Nurul Izzah MOHD-AZAMI

Universiti Malaya

Shih-Keng LOONG

Universiti Malaya https://orcid.org/0000-0001-7511-860X

Jing-Jing KHOO

Universiti Malaya

Norhidayu SAHIMIN

Universiti Malaya

Fang-Shiang LIM

Universiti Malaya

Nurul Aini HUSIN

Universiti Malaya

Nur Hidayana MAHFODZ

Universiti Malaya

Farah Shafawati MOHD-TAIB

Universiti Kebangsaan Malaysia

Siti Nabilah ISHAK

Universiti Kebangsaan Malaysia

Benjamin L. MAKEPEACE

University of Liverpool

Sazaly ABUBAKAR ( $\sim$ sazaly@um.edu.my)

Universiti Malaya

\section{Short Report}

Keywords: Infectious disease, Malaysia, rat bocavirus, Rattus sp., rodent

Posted Date: February 10th, 2022

DOI: https://doi.org/10.21203/rs.3.rs-1345217/v1

License: (9) This work is licensed under a Creative Commons Attribution 4.0 International License.

Read Full License 


\section{Abstract}

Rat (RBoV) and rodent (RoBoV) bocaviruses have previously been detected in Rattus norvegicus, however these viruses have not been reported in the rodent populations of Malaysia. We investigated the presence of RBoV and RoBoV in a cohort of archived rodent specimens. DNA barcoding of the rodent cytochrome $\mathrm{C}$ oxidase gene identified five different species, including; Rattus tanezumi R3 mitotype, Rattus tiomanicus, Rattus exulans, Rattus argentiventer and Rattus tanezumi sensu stricto. Three spleens were positive for RBoV $(1.84 \% ; 3 / 163)$ and no RoBoV was detected. Phylogenetic analyses of the partial non-structural protein 1 gene grouped the Malaysian RBoV strains together with RBoV strains from China. Further study among rats in different geographical locations hence is warranted for this relatively new virus.

\section{Text}

Human bocavirus (HBoV) was first reported in Sweden more than a decade ago [2]. Prior to the discovery, clinicians were baffled by cases of respiratory tract illness with apparently obscure etiology. Since then, $\mathrm{HBoV}$ is recognized as a pathogen that can cause nosocomial infection, especially amongst paediatric patients [6]. Other phylogenetically distinct bocaviruses have also been found in animals such as rodents [16], dogs [13], cats [1] and pigs [8]. Rodents are synanthropic small mammals that can easily adapt to areas close to human habitations [9]. Recently, HBoV was detected in the faeces of Rattus norvegicus in China [15]. The researchers found that the virus was closely related to the HBoV-2 strain. This finding alarmed scientists on the potential of rats serving as the carrier of HBoV and transmitting the virus to humans. Subsequently, Lau, Yeung [7] reported the detection of a novel rat bocavirus (RBoV) among $R$. norvegicus in China. A separate study in China did not detect RBoV, however, rodent bocaviruses (RoBoV) were instead detected [16]. Interestingly, the RoBoV strains were found to be more closely related to porcine bocaviruses as compared to RBoV in the phylogenetic analyses [14]. These findings showed that bocaviruses have established a niche in rodents and they (RBoV and RoBoV) are markedly different than HBoVs. Research efforts on RBoV and RoBoV are largely concentrated in China. There is however, no information on the prevalence of these bocaviruses in the population of rodents in Malaysia. Hence, the present study was conducted to investigate the possible presence of RBoV and RoBoV among the rodent populations in Peninsular Malaysia.

Rodents from the sampling sites at UM Plantations Sdn. Bhd., Johore (N2.02916, E103.87076), and Kampung Tumbuh Hangat, Perak (N4.313903, E100.929009) were trapped at several different times between December 2018 and December 2019. UM Plantations Sdn. Bhd. is an oil palm plantation located in the southern state of Johore, whereas Kampung Tumbuh Hangat is a village in central Perak that is surrounded by oil palm plantations and paddy fields. This study received the animal ethics approval from the Universiti Malaya Institutional Animal Care and Use Committee (G8/23122019/11102019-01/R). Permission was also obtained from the Department of Orang Asli Development (JAKOA) (JHEOA.PP.30.052 Jld. 6 (19)) for conducting this study at Kampung Tumbuh Hangat, Perak. Upon morphological identification, the trapped rodents were euthanized, followed by harvesting and archiving of selected tissues. The present study utilized the blood clots $(n=147)$ and spleens $(n=163)$ from a total 
of 163 rodents. Blood clots from the remaining 16 rodents were insufficient for downstream experiments, therefore were not included. The extraction for genomic DNA followed the NucleoSpin Tissue extraction (Macherey-Nagel, Düren, Germany) protocol. Cytochrome c oxidase ( $\mathrm{CO}$ ) gene fragment of 726-bp [4] was amplified from each rodent for species group determination (Table 1). All sequences obtained were compared to those in GenBank using the BLAST tool (http://www.ncbi.nlm.nih.gov/BLAST).

Table 1

Primers utilised in detecting bocavirus and DNA barcoding.

\begin{tabular}{|c|c|c|c|c|c|}
\hline Species & Target & $\begin{array}{l}\text { Primer } \\
\text { name }\end{array}$ & Oligonucleotide sequence ( $\left.5^{\prime}-3^{\prime}\right)$ & $\begin{array}{l}\text { Amplicon } \\
\text { size }\end{array}$ & Reference \\
\hline \multirow[t]{2}{*}{ Rodent } & $\mathrm{COI}$ & BatL5310 $a, c$ & ACTTCTGGGTGTCCAAAGAATCA & $726 b p$ & \multirow[t]{2}{*}{ [4] } \\
\hline & & $R 6036 R^{b, c}$ & CCTACTCRGCCATTTTACCTATG & & \\
\hline \multirow{4}{*}{$\begin{array}{l}\text { Rodent } \\
\text { bocavirus }\end{array}$} & NS1 & PBNS1-F1a & CCCAGTACAGGAAAGACCAACC & & \multirow[t]{8}{*}[16]{} \\
\hline & & $\mathrm{PBNS} 2-\mathrm{R} 1^{\mathrm{b}}$ & GAAGGGCATAACTTAGCCAACG & & \\
\hline & & PBNS1-F2 ${ }^{\mathrm{a}, \mathrm{c}}$ & GTAAATCTATTCGGCAATGTGA & $413 \mathrm{bp}$ & \\
\hline & & $\begin{array}{l}\text { PBNS1- } \\
\text { R2 } 2^{b, c}\end{array}$ & CATGTAGTGCAGTATCCGTCCA & & \\
\hline \multirow[t]{4}{*}{$\begin{array}{l}\text { Rat } \\
\text { bocavirus }\end{array}$} & NS1 & $\begin{array}{l}\text { RBoV-HK- } \\
\text { F1 }\end{array}$ & CTACTGGGCATGCGAACGTA & & \\
\hline & & $\begin{array}{l}\text { RBoV-HK- } \\
\mathrm{R}^{\mathrm{b}}\end{array}$ & CAGTTGCCTGTTGGTGTGTG & & \\
\hline & & $\begin{array}{l}\text { RBoV-HK- } \\
\text { F2 }^{a, c}\end{array}$ & ACAGCAGACAAGCCAACCAA & $248 b p$ & \\
\hline & & $\begin{array}{l}\text { RBoV-HK- } \\
\text { R2 }{ }^{b, c}\end{array}$ & TGCATTGTCTTCTGGCTGTCT & & \\
\hline
\end{tabular}

Nested polymerase chain reaction (PCR) targeting the non-structural protein 1 (NS1) gene was performed for the detection of RBoV and RoBoV [16]. For the detection of RBoV, primers RBoV-HK-F1 and RBoV-HKR1 were used for the first round of the nested PCR while, primers RBoV-HK-F2 and RBoV-HK-R2 were used for the second round of the nested PCR, resulting in the amplification of a 248-bp amplicon (Table 1). For the detection of RoBoV, primers PBNS1-F1 and PBNS2-R1 were used for the first round of the nested PCR while, primers PBNS1-F2 and PBNS1-R2 were used for the second round of the nested PCR, to amplify a 413-bp amplicon (Table 1). Purified amplicons were sequenced in both directions and the obtained sequences were compared to that available in the GenBank using the BLAST tool. A phylogenetic tree 
applying the maximum-likelihood method was then constructed to determine the genetic relationships of the bocaviruses obtained in this study with other representative bocaviruses obtained from the GenBank.

Analyses of the $\mathrm{CO} /$ sequences revealed that there were five groups of rat species identified in this study, comprising Rattus tanezumi sensu stricto, Rattus tiomanicus, Rattus tanezumi R3 mitotype, Rattus exulans and Rattus argentiventer. The COI sequences were deposited in the Barcode of Life Data System (BOLD) database under the following process IDs; UMNPA004-20 - UMNPA056-20 and UMNPA058-20 UMNPA068-20 for rodents captured from Johore, and UMNPA069-20, UMNPA071-20 - UMNPA076-20, UMNPA078-20 - UMNPA080-20, UMNPA082-20 - UMNPA083-20, UMNPA085-20, UMNPA087-20 UMNPA091-20, UMNPA093-20 - UMNPA102-20, UMNPA161-20 - UMNPA194-20, UMNPA196-20 UMNPA216-20 and UMNPA218-20 - UMNPA223-20 for rodents captured from Perak. The predominant species was the $R$. tanezumi $\mathrm{R} 3$ mitotype $(\mathrm{n}=116)$ followed by $R$. tiomanicus $(\mathrm{n}=22), R$. exulans $(\mathrm{n}=13), R$. argentiventer $(\mathrm{n}=11)$ and $R$. tanezumi s. $s .(\mathrm{n}=1)$ (Table 2$)$. RBoV was detected in three $R$. tanezumi $\mathrm{R} 3$ mitotype spleen samples. No RBoV was detected in the blood clots of all the studied rats. The RBoV positive rats were captured from Perak. No RBoV was detected in rats trapped in Johore and no RoBoV was detected from rats trapped at either site. The phylogenetic tree generated for RBoV using the NS1 gene showed that all the RBoV strains are grouped together and are separated from the other bocaviruses (Fig. 1). The Malaysian strains were distinctively different since they clustered into one monophyletic group next to the RBoV strains from China (Accession No. MG905222 and KT454514). Based on Table 3, the pairwise genetic distance showed that the Malaysian RBoV strains were similar to each other, while markedly different to the RBoV strains from China since the interspecific distances were more than $3 \%$ (the genetic distance among the Malaysian RBoV strains).

Table 2

The number of rats trapped in Perak and Johore.

\begin{tabular}{|llll|}
\hline \multirow{2}{*}{ Species } & \multicolumn{2}{l}{ Trapping Site } & Total number of individuals \\
\cline { 2 - 3 } & Perak $(\mathbf{n})$ & Johor $(\mathbf{n})$ & \\
\hline Rattus tanezumi R3 mitotype & 64 & 52 & 116 \\
\hline Rattus tiomanicus & 10 & 12 & 22 \\
\hline Rattus exulans & 10 & 3 & 13 \\
\hline Rattus tanezumi sensu stricto & 1 & 0 & 1 \\
\hline Rattus argentiventer & 11 & 0 & 11 \\
\hline Total number of individuals & 96 & 67 & 163 \\
\hline
\end{tabular}


Table 3

The pairwise distance generated by comparing the partial sequences of RBoV NS1 representatives to the sequences isolated from this study using MEGA11.

\begin{tabular}{|c|c|c|c|c|c|c|}
\hline No. & Name of sequence & 1 & 2 & 3 & 4 & 5 \\
\hline 1. & Rat bocavirus strain HK2S & & & & & \\
\hline 2. & Rat bocavirus strain XM.FA.63 & $2.03 \%$ & & & & \\
\hline 3. & UM-SNI01 & $31.08 \%$ & $31.92 \%$ & & & \\
\hline 4. & UM-SNI02 & $27.85 \%$ & $28.62 \%$ & $2.99 \%$ & & \\
\hline 5. & UM-SNIO3 & $28.64 \%$ & $29.45 \%$ & $1.97 \%$ & $2.00 \%$ & \\
\hline
\end{tabular}

To the best of our knowledge, only cases of HBoV [3] and porcine bocavirus [10] have been reported in Malaysia. Prior to the present study, there have been no published data on the prevalence of RBoV nor RoBoV in Malaysia. Since human infections caused by HBoV have been attracting considerable attention in recent years due to its possible zoonotic origin $[2,10,12]$, we sought to examine the baseline levels of RBoV and RoBoV in a cohort of archived rodent specimens.

As bocaviruses are respiratory-associated pathogens, their presence would more likely be detected in respiratory tissues. However, the rodent lung tissues in our archive have been dedicated for another study. As such, the spleens and blood clots were selected instead. HBoVs have been detected in the human patient blood [11] and RBoV in the spleens of rats [7], making these biological samples feasible targets. Interestingly, RBoV was detected only from the spleen of the $R$. tanezumi R3 mitotype in this study and none were detected in the blood clots. Detection of RBoV was reported in the throat swabs and faeces of rodents captured from four provinces in China [14]. Besides that, RBoV has been detected in the alimentary and kidney tissues [7]. These findings suggest that RBoV could reside in the alimentary and respiratory tracts, translocate into the kidney and spleen, followed by secretion in the faeces. A study reported by Zhang, Song [16] discovered RoBoV in the lungs of seven different rodent species namely; $R$. norvegicus, Mus musculus, Apodemus agrarius, Cricetulus barabensis, Rattus flavipectus, Rattus rattus and Rhombomys opimus. Further genetic analyses revealed that RoBoV shares relatively low amino acid similarity of about $51 \%$ when compared to RBoV and is more closely related to porcine bocavirus [16]. Given that RoBoV was not found in any rodent samples in this study, it could imply that RoBoV is not present in rodents at the sampling sites.

All three RBoV strains detected in the present study were clustered in a sister clade next to the RBoVs from China (Fig. 1), indicating that the Malaysian RBoV strains are genetically different as compared to those from China. RoBoV and RBoV strains, reported respectively by Zhang, Song [16] and Lau, Yeung [7], were detected in urban cities while our study sites are transition areas such as the oil palm plantations and paddy fields, depicting the difference in habitat. The low RBoV detection rate of $1.84 \%(3 / 163)$ could be explained by the difference in the rat population density between the study sites (oil palm 
plantation/paddy fields vs urban cities). The study sites are spacious and uncrowded, unlike the condensed urban cities [17], resulting in less contact between the rats and potentially lower transmission of RBoV. Urban rats have to compete with each other for resources and the increase in contact could contribute to the transmission of respiratory pathogens such as RBoV [5].

All the RBoV positive strains were detected from the $R$. tanezumi $\mathrm{R} 3$ mitotype, suggesting its potential as the carrier of RBoV. However, we cannot conclude that RBoV is exclusively found in R. tanezumi R3 mitotype from just one relatively small-scale study. Moreover, data on bocavirus among rodents is lacking, especially in Southeast Asia. Thus, more studies that include a larger sample size covering various locations have to be conducted to gain better insights into the prevalence of RBoV in Malaysia. In many instances, we found unspecific binding in the nucleic acid amplification for RBoV. This suggests that the primers might not be specific enough to target Malaysian RBoV strains and this was somewhat expected since the primers were designed based on RBoV strains from China [16]. Consequently, this would also imply that we could be underestimating the RBoV detection rate in this study.

In conclusion, we report here the detection of RBoV in the R. tanezumi R3 mitotype captured in Malaysia. Further surveillance of RBoV in Malaysia and other countries in Southeast Asia is warranted since very little information is available on the biology, pathology and transmission of this relatively new virus.

\section{Declarations}

\section{Conflict of interest}

The authors declare that they have no conflict of interest.

\section{Acknowledgements}

We acknowledge the funding from the Ministry of Higher Education, Malaysia for niche area research under the Higher Institution Centre of Excellence (HICoE) program (MO002-2019). This research was also supported by an Institutional Links grant, ID 332192305, under the Newton-Ungku Omar Fund partnership. The grant was funded by the UK Department of Business, Energy and Industrial Strategy

(BEIS) and the Malaysian Industry-Government Group for High Technology (MIGHT), and delivered by the British Council.

\section{References}

1. Abayli, H. and Can-Sahna, K. 2021. First detection of feline bocaparvovirus 2 and feline chaphamaparvovirus in healthy cats in Turkey. Vet. Res. Commun. 1-10. doi:10.1007/s11259-02109836-w. 
2. Allander, T., Tammi, M. T., Eriksson, M., Bjerkner, A., Tiveljung-Lindell, A. and Andersson, B. 2005. Cloning of a human parvovirus by molecular screening of respiratory tract samples. Proc. Natl. Acad. Sci. 102: 12891-12896. doi:10.1073/pnas.0504666102.

3. Etemadi, M., Azizi Jalilian, F., Wahab, N., Jahanshiri, F., Amini, R., Othman, N. and Sekawi, Z. 2012. First detected human bocavirus in a Malaysian child with pneumonia and pre-existing asthma: $A$ case report. Int. Med. J. Malays. 67: 433-434.

4. Herbreteau, V., Jittapalapong, S., Rerkamnuaychoke, W., Chaval, Y., Cosson, J. F. and Morand, S. 2011. Protocols for field and laboratory rodent studies.

http://www.ceropath.org/FichiersComplementaires/Herbreteau_Rodents_protocols_2011.pdf. [accessed on Dec 31, 2021].

5. Judson, S. D. and Munster, V. J. 2019. Nosocomial transmission of emerging viruses via aerosolgenerating medical procedures. Viruses. 11: 940. doi:10.3390/v11100940.

6. Kobayashi, H., Shinjoh, M., Sudo, K., Kato, S., Morozumi, M., Koinuma, G., Takahashi, T., Takano, Y., Tamura, Y. and Hasegawa, N. 2019. Nosocomial infection by human bocavirus and human rhinovirus among paediatric patients with respiratory risks. J. Hosp. Infect. 103: 341-348. doi:10.1016/j.jhin.2019.05.002.

7. Lau, S. K. P., Yeung, H. C., Li, K. S. M., Lam, C. S. F., Cai, J. P., Yuen, M. C., Wang, M., Zheng, B. J., Woo, P. C. Y. and Yuen, K. Y. 2017. Identification and genomic characterization of a novel rat bocavirus from brown rats in China. Infect. Genet. Evol. 47: 68-76. doi:10.1016/j.meegid.2016.11.014.

8. Lee, C., Tan, C., Tan, Y., Wong, J., Jacob, D., Lim, J., Ananthan, G., Arshad, S., Selvarajah, G. and Ooi, P. 2019. First nearly complete genome sequence of porcine bocavirus strain from Malaysia. Adv. Anim. Vet. Sci. 7: 776-781. doi:10.17582/journal.aavs/2019/7.9.776.781.

9. Loong, S. K., Che-Mat-Seri, N. A. A., Tan, K. K., Azizan, N. S., Ahmad-Nasrah, S., Mohd-Zain, S. and AbuBakar, S. 2021. Isolation of Kytococcus schroeteri from the brown rat Rattus norvegicus. Asian Pac. J. Trop. Med. 14: 191-192. doi:10.4103/1995-7645.312515.

10. Mohan Jacob, D., Lee, C. Y., Arshad, S. S., Selvarajah, G. T., Bande, F., Ong, B. L. and Ooi, P. T. 2018. First molecular detection of porcine bocavirus in Malaysia. Trop. Anim. Health Prod. 50: 733-739. doi:10.1007/s11250-017-1489-z.

11. Nora-Krukle, Z., Vilmane, A., Xu, M., Rasa, S., Ziemele, I., Silina, E., Söderlund-Venermo, M., Gardovska, D. and Murovska, M. 2018. Human bocavirus infection markers in peripheral blood and stool samples of children with acute gastroenteritis. Viruses. 10: 639. doi:10.3390/v10110639.

12. Schildgen, 0. 2013. Human bocavirus: Lessons learned to date. Pathogens. 2: 1-12. doi:10.3390/pathogens2010001.

13. Wang, Y., Li, W., Guo, X., Zhang, D., Sun, J., Fu, Z., Liu, G., Li, Y. and Jiang, S. 2021. Development of SYBR Green I-based polymerase chain reaction for feline bocavirus 1 detection. 3 Biotech. 11: 1-6. doi:10.1007/s13205-020-02577-8.

14. Xiong, Y. Q., Zhou, J. H., Zhang, M. Y., You, F. F., Li, D. L. and Chen, Q. 2018. Presence of rat bocavirus in oropharyngeal and fecal samples from murine rodents in China. Arch. Virol. 163: 3099-3103. 
doi:10.1007/s00705-018-3943-2.

15. You, F. F., Zhang, M. Y., Wu, F., Li, Q. S. and Chen, Q. 2022. Human bocavirus 2 detected in Rattus norvegicus feces in China. Arch. Virol. 167: 171-175. doi:10.1007/s00705-021-05274-w.

16. Zhang, C., Song, F., Xiu, L., Liu, Y., Yang, J., Yao, L. and Peng, J. 2018. Identification and characterization of a novel rodent bocavirus from different rodent species in China. Emerg. Microbes Infect. 7: 1-11. doi:10.1038/s41426-018-0052-y.

17. Zünd, D. and Bettencourt, L. M. A. 2019. Growth and development in prefecture-level cities in China. PLoS One. 14: e0221017. doi:10.1371/journal.pone.0221017.

\section{Figures}

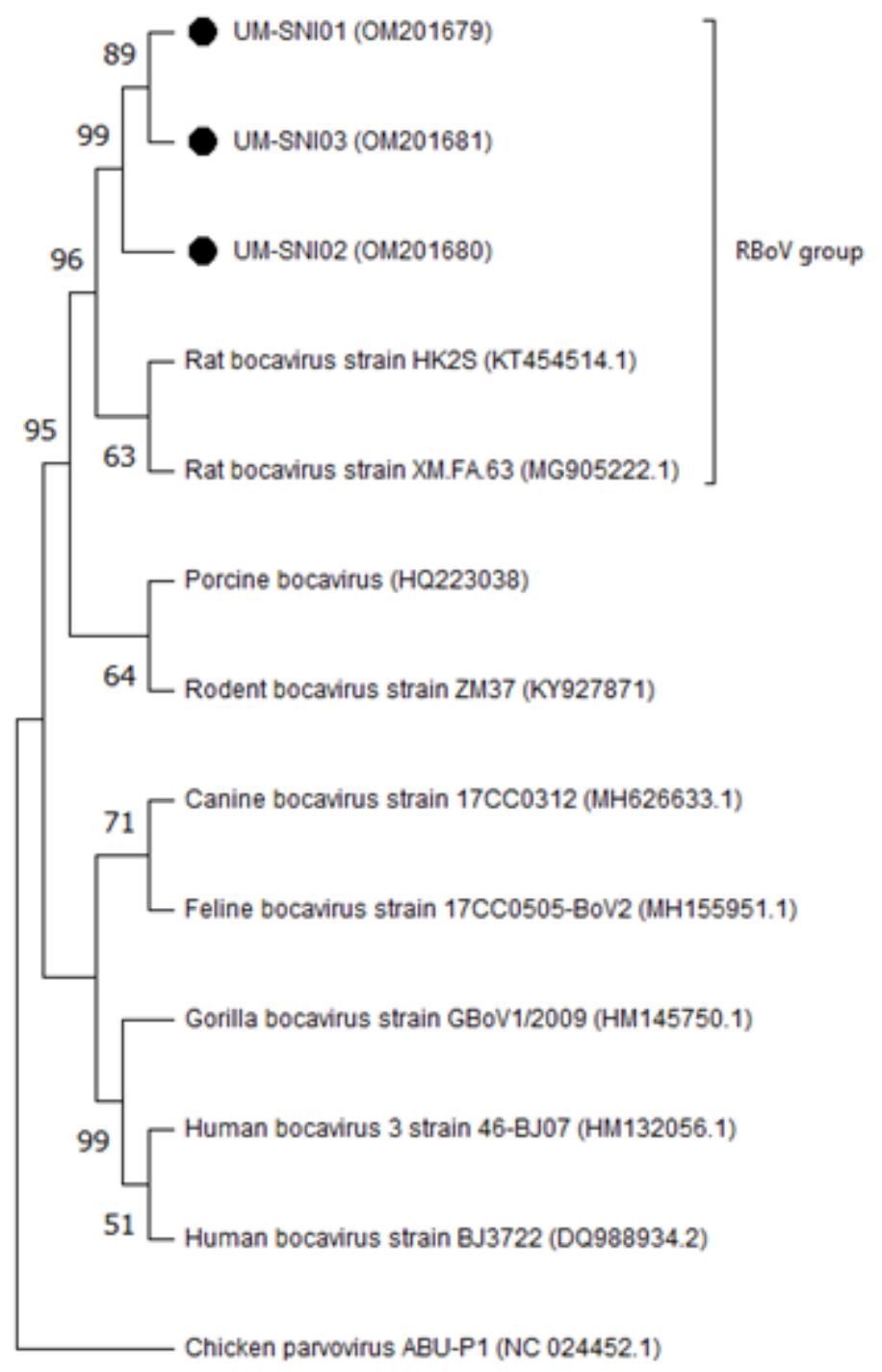

\section{Figure 1}

Phylogenetic tree of the partial NS1 sequences of RBoV detected from the rats in this study. Other bocavirus representatives were obtained from the GenBank with respective accession numbers in 
parentheses. Black bullets represent the RBoV strains detected from spleen samples in the present study. The tree was inferred using the maximum likelihood method implemented in MEGA11. 